\title{
Protective antibody and cytokine responses in mice following immunization with recombinant beta-tubulin and subsequent Trypanosoma evansi challenge
}

\author{
Anup Kumar Tewari ${ }^{1,2^{*}+} \mathbb{D}$, Samarchith P. Kurup ${ }^{2,3 \dagger}$, Surajit Baidya ${ }^{2,5}$, John R. Barta ${ }^{1}$ and Bhaskar Sharma ${ }^{4}$
}

\begin{abstract}
Background: Trypanosomosis or Surra, caused by the flagellated hemoprotozoan parasite Trypanosoma evansi, is a disease of economic importance through its wide prevalence in domestic livestock in tropical countries. In the absence of a protective vaccine, management of the disease relies on a few available chemotherapeutic agents. Although humoral immunity is the mainstay of resistance to T. evansi, the ability of the parasite to vary its immunodominant surface proteins to subvert the immune system has forced vaccine efforts to target a variety of invariant epitopes. Beta tubulin, an integral component of the trypanosome cytoskeleton, was therefore targeted using the recombinant form of the protein for immunization.
\end{abstract}

Methods: The 1329 bp coding sequence of beta tubulin gene was PCR amplified and cloned in PQETriSystem expression vector. Recombinant beta tubulin was heterologously expressed in Escherichia coli as a $46 \mathrm{KDa}$ fusion protein and used for immunization of mice. The Ig response was studied by ELISA, whereas the cytokine response was measured using a cytometric bead-based assay quantified by flow cytometry.

Result: Immunization with recombinant beta ( $\beta$ )-tubulin protein induced a beta-tubulin specific humoral immune response of predominantly lgG2a isotype. Lethal challenge with $T$. evansi blood-form trypomastigotes post-immunization elicited a robust anamnestic response. An abundance of IFN- $\gamma$ further confirmed the Th-1 bias of the protective response. We also observed extended survival and better control of the challenge infection in the immunized mice.

Conclusions: A robust anamnestic response following challenge including a Th-1 serum cytokine profile coupled with increased survival is indicative of protective immunity in the immunized mice. These observations suggest that $\beta$-tubulin of $T$. evansi is a viable antigenic target for development of a vaccine against this important livestock pathogen.

Keywords: Recombinant beta tubulin, Cytokine, Immune response, Surra, Trypanosoma evansi

\footnotetext{
* Correspondence: atewari@uoguelph.ca

${ }^{\dagger}$ Equal contributors

'Department of Pathobiology, Ontario Veterinary College, University of

Guelph, Guelph, ON N1G 2W1, Canada

${ }^{2}$ Division of Parasitology, Indian Veterinary Research Institute, Izatnagar, Uttar

Pradesh 243 122, India

Full list of author information is available at the end of the article
}

\section{() Biomed Central}

(c) 2015 Tewari et al. Open Access This article is distributed under the terms of the Creative Commons Attribution 4.0 International License (http://creativecommons.org/licenses/by/4.0/), which permits unrestricted use, distribution, and reproduction in any medium, provided you give appropriate credit to the original author(s) and the source, provide a link to the Creative Commons license, and indicate if changes were made. The Creative Commons Public Domain Dedication waiver (http://creativecommons.org/publicdomain/zero/1.0/) applies to the data made available in this article, unless otherwise stated. 


\section{Background}

Trypanosoma evansi, a unicellular hemoflagellate, is the causative agent of 'surra', a debilitating disease of a wide range of livestock species in the tropics. This parasite is transmitted mechanically among its wide range of susceptible hosts by several species of hematophagous flies in Asia, Africa, Latin America and parts of Europe [1-4]. The progression and outcome of infections varies widely depending on the host species; fever, progressive anemia or corneal opacity is typical in companion animals whereas farm animals suffer from loss of condition, productivity and draught efficiency, neurological abnormalities, abortion or immunosuppression [5-8].

Management of surra is based chiefly on chemotherapy. However, limited therapeutic options and the development of drug-resistance have posed a major threat to our abilities to contain the disease. The need for a safe and cost-effective vaccine against surra [9] makes identification of protective epitopes a priority. Though T. evansi has an abundance of highly immunogenic glycoproteins presented on its surface (variable surface glycoprotein, VSG), the active and systematic variation of these surface antigens during the course of an infection has limited the prospects of targeting them for vaccine development [10]. Consequently, immunization strategies in surra have focused on various alternate, invariant, and often immunologically subdominant epitopes [11-14].

Intracellular antigens can act as protective epitopes in parasitic infections including malaria [15], schistosomosis [16] and trypanosomosis [9, 10, 12] although the underlying mechanisms have not been described. One promising intracellular antigen of trypanosomes is beta ( $\beta$ ) tubulin which belongs to the large tubulin protein family. Unlike in higher vertebrates and other invertebrates that possess orthologues of $\beta$ tubulin with diverse and dissimilar structural and functional attributes, trypanosomes have a single isoform in the Trypanosomatidae [10]. This protein is an important structural component of the flagellum and is critical for structural stability and replication making it a promising therapeutic and vaccine target in protozoa [9]. Immunization with native, recombinant or cDNA encoded trypanosomatid tubulin has shown varying levels of protection against different African trypanosomes in experimental surra $[9,10,17]$.

Humoral immunity has long known to be important in protection against African trypanosomes. Induction of a rapid B-cell activation $[18,19]$ accompanied by a $\mathrm{T}$ helper type (Th) 1 response with production of proinflammatory cytokines is characteristic of successful immunological control of acute African trypanosomosis in the host [20-23] . A fine balance between the proinflammatory and anti-inflammatory cytokines that effectively controls the parasitemia [24] and associated anemia [25] in newly acquired infections are thought to be responsible for trypanotolerance. In the present study, we characterized the humoral antibody responses and associated Th1-polarized serum cytokine profiles in mice following immunization with recombinant $T$. evansi $\beta$-tubulin that provided enhanced survival of mice in the face of a lethal challenge infection.

\section{Methods}

\section{Mice}

Female Swiss albino mice (6-8 weeks) were procured from the Laboratory Animal Research (LAR) Division, Indian Veterinary Research Institute (IVRI), Izatnagar. The mice were maintained under standard feeding and rearing conditions at the lab animal housing facility of the Division of Parasitology, IVRI, Izatnagar.

\section{Recombinant expression-vector}

The entire open reading frame (ORF) of $T$. evansi $\beta$ tubulin (EU483116) was amplified by PCR from $T$. evansi cDNA template with primers, $\mathrm{F}_{1} \mathrm{X}$ : $5^{\prime}$-AGAA TTCCATGGGTGAGATTGTGTGCGT-3' containing

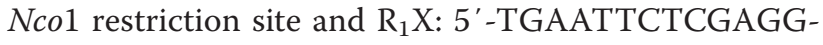
TATTGCTCCTCCTCGTC-3' containing the Xho1 restriction site. The purified PCR product was digested with Nco1 and Xho1 and cloned into pQE TriSystem plasmid vector (Qiagen, CA, USA) to give $\mathrm{pQE}-\mathrm{Te}-\beta$ tubulin recombinant plasmid.

\section{Expression of rTe- $\beta$-tubulin}

Recombinant $\mathrm{pQE}-T e-\beta$-tubulin construct was used for expression of $T e-\beta$-tubulin in $E$. coli as per the manufacturer's protocol (Qiagen, CA, USA). In short, $100 \mu \mathrm{l}$ of overnight culture of pQE-Te- $\beta$-tubulin transformed M15 strain $E$. coli was transferred into $50 \mathrm{ml}$ of $\mathrm{LB}$ broth (Thermo Scientific, MA, USA) containing $100 \mu \mathrm{g} / \mathrm{ml}$ ampicillin and was allowed to grow in a shaking incubator at $37{ }^{\circ} \mathrm{C}$ until mid-log phase $(\sim 2 \mathrm{~h})$ after which isopropyl-beta-D-thiogalactopyranoside (IPTG) (Thermo Scientific, MA, USA) was added at $1 \mathrm{mM}$ final concentration to induce expression. The culture was maintained for up to $6 \mathrm{~h}$ post induction. rTe- $\beta$-tubulin produced in the $E$. coli cell lysate screened using SDSPAGE. The identity of the expressed protein was confirmed by western blot probed with anti-histidine (His) tag antibody (Invitrogen, CA, USA). The His-tagged rTe- $\beta$-tubulin present in the insoluble pellet was solubilized under denaturing conditions with $8 \mathrm{M}$ urea, and then purified using Ni-NTA agarose column (Qiagen, $\mathrm{CA}$, USA), renatured by dialysis (MWCO $10 \mathrm{~K}$, Thermo Scientific, MA, USA) against PBS, $\mathrm{pH} 7.4$, at $4{ }^{\circ} \mathrm{C}$ for $36 \mathrm{~h}$ and its purity determined by SDS-PAGE and stored at $-20{ }^{\circ} \mathrm{C}$ until use. The concentration of $\mathrm{rTe}-\beta$-tubulin was determined with modified Lowry protein assay kit 
(Thermo Scientific, MA, USA) as per the manufacturer's protocol.

\section{Immunization and challenge}

Female Swiss albino mice (6-8 weeks) were immunized subcutaneously with $50 \mu \mathrm{g}$ of $\mathrm{rTe}-\beta$-tubulin in Freund's complete adjuvant (FCA) or with FCA alone as control. Similar booster dosages were administered 21 days later, but with incomplete Freund's adjuvant (IFA). At 35 days post primary immunization, all mice were challenged intraperitoneally with $10^{3} \mathrm{~T}$. evansi blood form trypomastigotes.

\section{Measurement of specific antibody response}

The serum antibody response was studied by enzyme linked immunosorbent assay (ELISA). A standard 96 well ELISA plate (Nunc, NY, USA) was coated for $1 \mathrm{~h}$ at $37{ }^{\circ} \mathrm{C}$ with the $\mathrm{rTe}-\beta$-tubulin $(5 \mu \mathrm{g} / \mathrm{ml}$ dissolved in $50 \mathrm{mM}$ sodium carbonate coating buffer, $\mathrm{pH}$ 9.6) followed by overnight blocking at $4{ }^{\circ} \mathrm{C}$ with $5 \%$ bovine serum albumin in PBS. After washing, $100 \mu$ of heat inactivated serum $\left(56{ }^{\circ} \mathrm{C}\right.$ for $30 \mathrm{~min}$ ) from immunized or naive mice was added at 1:50 dilution. After incubating for $2 \mathrm{~h}$ at $37{ }^{\circ} \mathrm{C}$, sera samples were removed, the wells washed and $100 \mu \mathrm{l}$ of HRPase conjugated goat anti-mouse IgG (Bangalore Genie, Karnataka, India) or IgG2a (Santa Cruz, CA, USA) added at 1:5000 dilution to the wells and incubated for $2 \mathrm{~h}$ at $37{ }^{\circ} \mathrm{C}$. Washed plates were developed for 15 mins at RT, using $100 \mu \mathrm{l} /$ well of OPD substrate $(15 \mu \mathrm{g} / \mathrm{ml}$ in citrate phosphate buffer, $\mathrm{pH}$ 5.0). The reaction was stopped by the addition of $50 \mu \mathrm{l} 2 \mathrm{M}$ sulfuric acid and the plates were read at $\mathrm{OD}_{492 \mathrm{~nm}}$ in a micro plate reader (Tecan, Mannedorf, Switzerland).

\section{Measurement of serum cytokines}

The concentration of Interleukin (IL)-4, IL-5, IL -10, Interferon (IFN) $-\gamma$ and Tumor Necrosis Factor (TNF)- $\alpha$ were determined in the serum of mice every $24 \mathrm{~h}$ until 6 days post T. evansi challenge using the BD mouse Th1/Th2 cytokine CBA Kit (BD Biosciences, CA, USA) following the manufacturer's protocol. Briefly, $50 \mu \mathrm{l}$ of diluted serum sample separated from blood collected every $24 \mathrm{~h}$ post challenge, until 6 days was mixed with $50 \mu \mathrm{l}$ of the mixed capture beads and $50 \mu \mathrm{l}$ of the mouse Th1/Th2 PE detection reagent. The tubes were incubated at room temperature for $2 \mathrm{~h}$ in the dark, followed by a wash. The samples were subsequently resuspended in $300 \mu \mathrm{l}$ of wash buffer before acquisition on the FACS Calibur Flow Cytometer (BD Biosciences, CA, USA) and analyzed using the FCAP Array Software (BD Biosciences). Standard curves were generated for each cytokine using the mixed bead standard provided with the kit and were used to determine the concentration of each cytokines in the serum samples. Serum IL-10 level was determined using an ELISA based kit (Thermo Scientific, MA, USA) following the manufacturer's protocol.

\section{Determination of parasitemia}

The parasitemia in mice following $T$. evansi challenge was monitored microscopically every $24 \mathrm{~h}$ from day 1 until 11 post infection using a standardized dilution of blood obtained from tail bleeding. Counts are expressed as trypomastigotes per $40 \times$ microscopic field and data are presented as means for each time point.

\section{Statistical analysis}

Data are expressed as mean \pm standard error of mean (s.e.m) and are derived from at least triplicate observations per sample per time-point. Results were analyzed by Student $t$-test and differences were considered significant if the $p$-value was $\leq 0.05$.

\section{Ethics statement}

All animal experimentation was conducted in compliance with the ethical considerations and guidelines issued by the Committee for the Purpose of Control and Supervision of Experiments on Animals (CPCSEA) of the Government of India and with the approval of the Institutional Animal Ethics Committee (IAEC, IVRI) regarding laboratory animals.

\section{Results}

Heterologous expression and purification of Te- $\beta$-tubulin To generate $\mathrm{rTe}$ - $\beta$-tubulin, the amplified $T e$ - $\beta$-tubulin gene (Fig. 1a) from a cDNA template of T. evansi trypomastigote stage was first cloned into the expression inducible $\mathrm{pQE}$-TriSystem vector. Escherichia coli transformed with this construct expressed $\mathrm{rTe}$ - $\beta$-tubulin that was purified by affinity column chromatography (Fig. 1b). The identity of expressed $\mathrm{rTe}$ - $\beta$-tubulin protein was confirmed by Western blot (Fig. 1c).

\section{Serum cytokine and antibody responses following immunization}

To determine cytokine responses following vaccination and subsequent specific humoral immune response mounted against $\mathrm{rTe}-\beta$-tubulin, sera from mice inoculated with $\mathrm{rTe}$ - $\beta$-tubulin $(50 \mu \mathrm{g})$ in FCA or FCA alone (control) were compared. At $24 \mathrm{~h}$ post inoculation, mice inoculated with $\mathrm{rTe}-\beta$-tubulin in FCA showed significantly lower IL-4 and significantly higher TNF- $\alpha$ levels in the serum compared to pre-immunization levels (Fig. 2a). IL-4 or TNF- $\alpha$ levels in mice inoculated with FCA alone did not differ significantly from the mice inoculated with $r T e-\beta$-tubulin in FCA (data not shown). At 14 days post inoculation, mice immunized with $\mathrm{rTe}-\beta$ tubulin had developed significantly higher titers of $T e-\beta$ tubulin-specific IgG antibodies (1.8 fold higher $\mathrm{OD}_{492}$ 


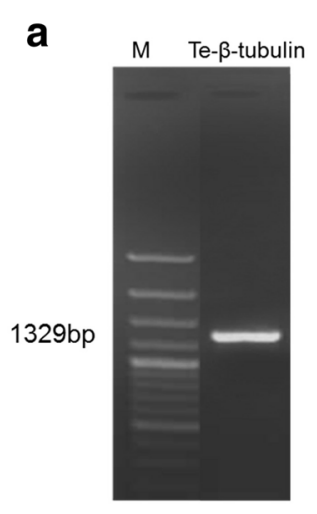

$\mathbf{C}$

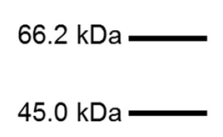

\section{b}

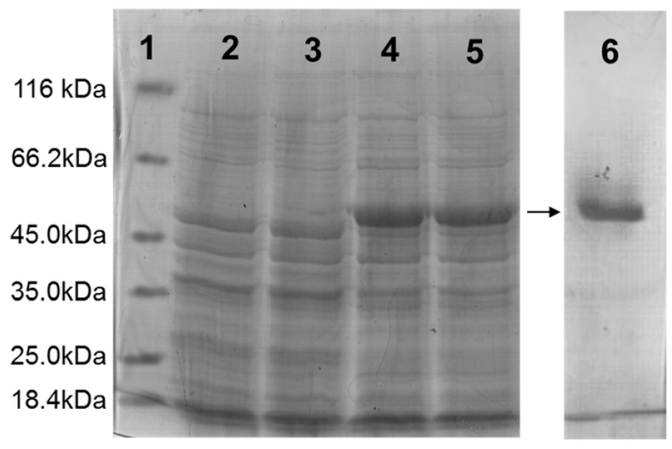

1

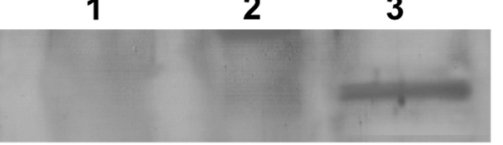

Fig. 1 Heterologous expression of recombinant $\beta$-tubulin from T. evansi. a PCR amplification of T. evansi $\beta$-tubulin gene. $\mathbf{b}$ Induced heterologous expression and purification of recombinant T. evansi $\beta$-tubulin protein ( $50 \mathrm{kDa}$ ) shown by SDS-PAGE. Protein molecular weight marker (Lane 1), pre-induction Te- $\beta$-tubulin transgenic E. coli lysate (lane 2), induced Te- $\beta$-tubulin transgenic E.coli lysate collected 2, 4,or 6 h post-induction (lanes 3-5, respectively) and affinity-purified, renatured rTe- $\beta$-tubulin (lane 6). c Identity of the expressed rTe- $\beta$-tubulin confirmed by Western blot using anti-His-tag antibody probing induced (4 h) Te- $\beta$-tubulin transgenic E. coli culture supernatant (lane 1), pre-induction (lane 2) or induced (4 h) (lane 3) Te- $\beta$-tubulin transgenic E. coli lysate

readings) compared to FCA controls; Te- $\beta$-tubulin-specific IgG2a titers in vaccinated mice were significantly higher (7.7 fold higher $\mathrm{OD}_{492}$ readings) than FCA control mice (Fig. 2b).

\section{Serum cytokine and antibody responses following $T$. evansi challenge}

Mice challenged with a lethal dose of live blood stage $T$. evansi 35 days post-immunization showed significantly higher $T e$ - $\beta$-tubulin specific (IgG) serum antibody titers compared to adjuvant-control or unimmunized mice at 4 days post-challenge (Fig. 3a). Mice inoculated with rTe- $\beta$-tubulin in FCA and challenged with $T$. evansi 35 days post-immunization had dramatically different kinetics of serum cytokine levels compared with mice inoculated with FCA only (Figs. 3b-f). Mice immunized with $\mathrm{rTe}-\beta$-tubulin demonstrated unchanging or slowly dropping serum IL-4 and IL-5 concentrations following challenge. Modest early (1-2 DPC) increases in the concentration of TNF- $\alpha$ and IL-10 were followed by slowly a

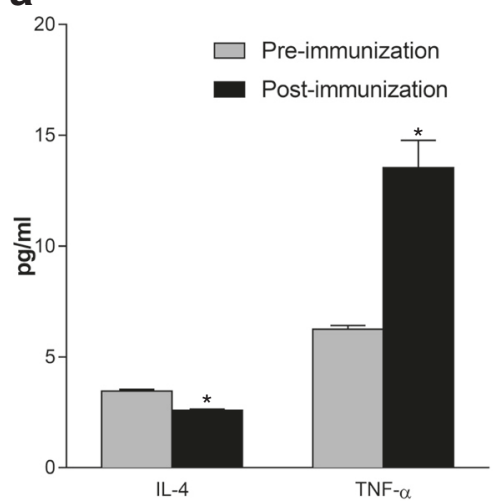

b

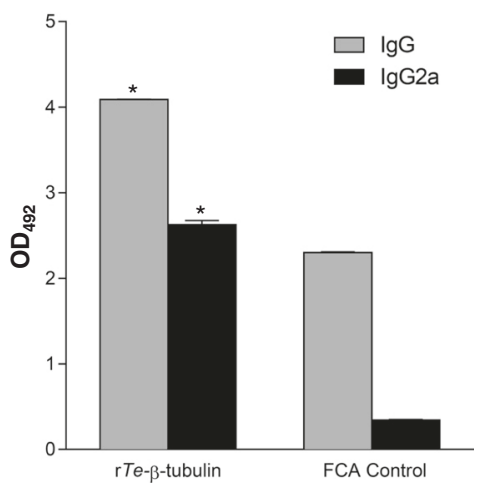

Fig. 2 Trypanosoma evansi $\beta$-tubulin-specific immune response induced post immunization with rTe- $\beta$-tubulin. a Serum levels of IL-4 and TNF-a in mice pre- and $24 \mathrm{~h}$ post-inoculation with FCA adjuvenated rTe- $\beta$-tubulin; asterisks indicate significant differences $(p \leq 0.05)$ between the pre- and post-immunization mean levels for each cytokine. $\mathbf{b}$ Te- $\beta$-tubulin-specific lgG and lgG2a antibody in mouse sera at 14 days post immunization with rTe- $\beta$-tubulin or FCA control. Data presented as mean \pm s.e.m. from at least 6 mice/treatment; asterisks indicate significant differences $(p \leq 0.05)$ between the mean $\mathrm{OD}_{492}$ values for serum lgG or lgG2a of FCA adjuvenated rTe- $\beta$-tubulin vaccinated mice compared with FCA-only inoculated control mice 


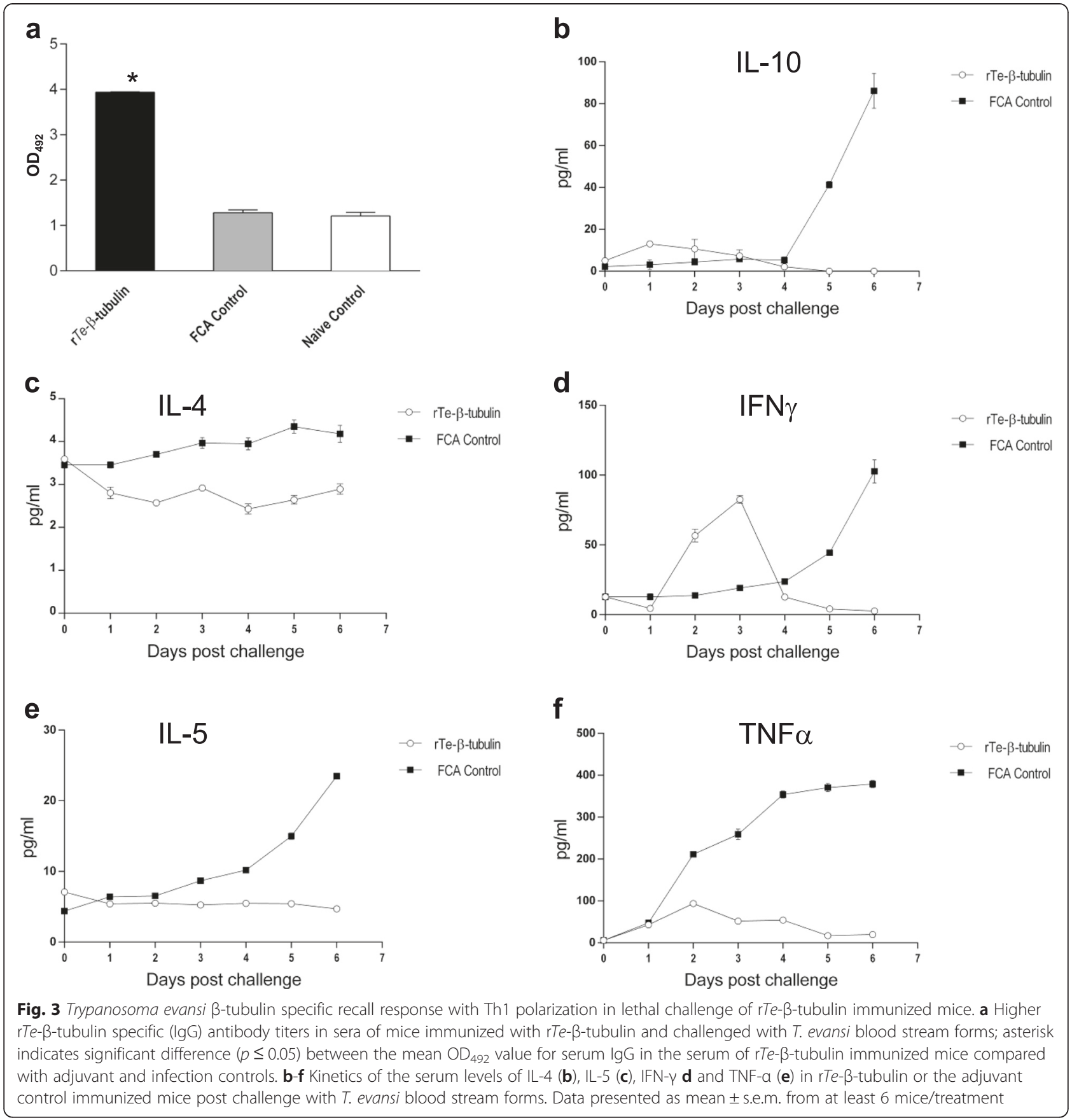

decreasing serum concentrations in mice inoculated with $r T e-\beta$-tubulin in FCA. In these same mice, interferon- $\gamma$ increased in concentration following challenge (peaking at $3 \mathrm{DPC}$ ) and then returned to lower levels for the remainder of the challenge period. In contrast, all measured cytokines increased from their levels at day of challenge until the end of the challenge period in mice inoculated with FCA only.

\section{rTe- $\beta$-tubulin immunization enhanced survival following}

\section{T. evansi challenge}

Mice immunized with $\mathrm{rTe}$ - $\beta$-tubulin exhibited a delayed appearance, as well as lower numbers, of T. evansi trypomastigotes in blood following challenge (Fig. 4a). All mice in the FCA only control group died by day 6 post challenge whereas all mice immunized with $\mathrm{rTe}$ - $\beta$-tubulin in FCA survived until at least day 10 post challenge. 


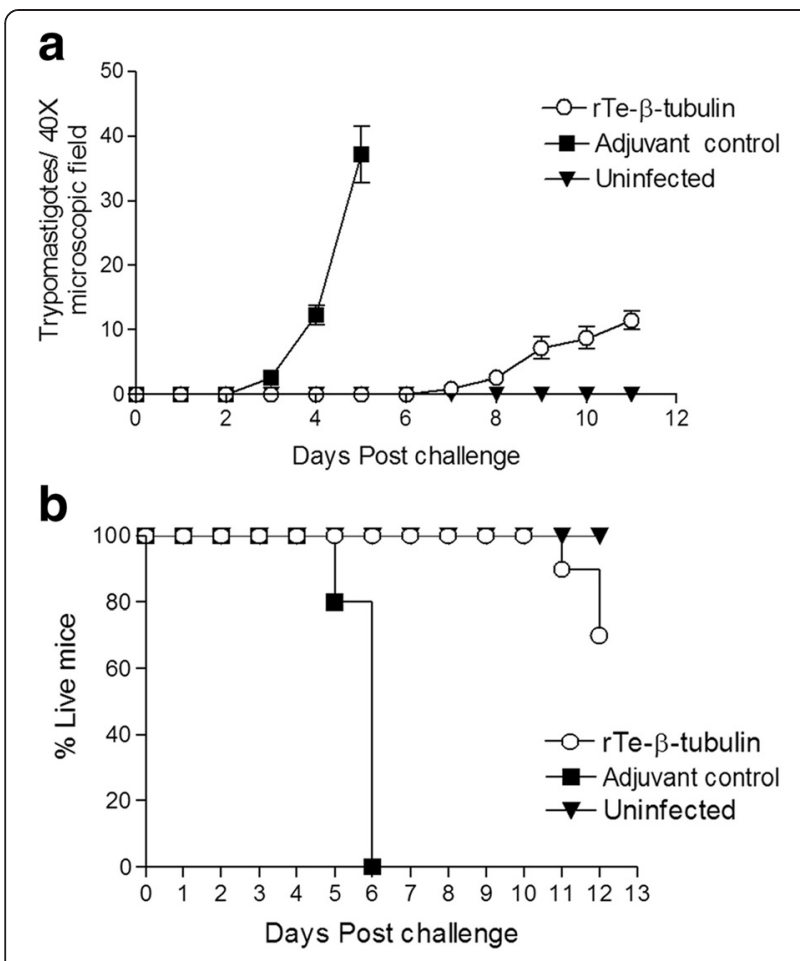

Fig. 4 Mice immunized with rTe- $\beta$-tubulin are better protected from a lethal challenge with $T$. evansi. The $r T$ - $\beta$-tubulin immunized mice demonstrated lower parasitemia (a) and prolonged survival (b) following a virulent $T$. evansi challenge compared with similarly challenged adjuvant only controls. Daily counts of bloodstream trypomastigotes represent means \pm s.e.m. from at least 10 mice per treatment

However, all the mice in the latter group eventually succumbed to infection by 28 days post challenge (Fig. 4b).

\section{Discussion}

Surra is an insidious disease complex caused by the unicellular, flagellated protozoan $T$. evansi that leads to the development of severe and often fatal anemia. The infection is prevalent in a wide range of domestic and wild animal species across the tropical and semitropical regions of Eurasia and Africa [8]. Although management of the disease by chemotherapy is feasible, its successful application is limited by the low sensitivity of conventional microscopy and serological tools that prevent the timely identification of chronic infections and carrier animals. The spread of the infection in susceptible animals often remains unchecked impacting the health and productivity of livestock in the tropics. The ability of $T$. evansi to vary its immuno-dominant major surface antigen to escape host immune responses limits the prospects of developing a conventional vaccine against $T$. evansi targeting epitopes typically exposed on the plasma membrane of the parasite [10]. Consequently, evaluation of internal antigens of $T$. evansi (e.g. molecules not typically exposed on the cell surface) for their ability to induce protective immune responses has become necessary [10, 17].

There have been multiple attempts at developing native or recombinant protein based vaccines against various protozoal infections but often with limited success compared vaccines based on whole organisms that better reflect the complexity of the target pathogen [26, 27]. Protective immunization against African trypanosomosis has been particularly challenging owing to the sequential expression of variable surface antigen molecules by the parasite that efficiently subverts the humoral immune responses [14, 28]. However, induction of protective immunity to various levels has been achieved by targeting invariable antigens on African trypanosomes; mice vaccinated with native tubulin purified from $T . b$. brucei were protected from $T$. $b$. brucei, T. congolense or T. b. rhodesiense infections [9]. Likewise, immunization with the microtubule associated protein (MAP) p52, GAPDH and MAP p15 protected mice from T. brucei challenge [29]. Recombinant protein [10,12] or DNA [17] based vaccines targeting the tubulin of various African trypanosomes have also provided partial protection.

Production of IgG2 antibodies and the activity of cytotoxic $\mathrm{T}$ cells are characteristics of Th1 immune response in mice, whereas a predominance of IgM, IgG1, IgA and IgE class antibodies represent Th2 response. In general, vaccines adjuvenated with FCA and delivered subcutaneously generate a Th1 rather than Th2 type immune response [30]. We observed significantly higher titers of $T$. evansi $\beta$ tubulin specific IgG antibody, specifically of IgG2a isotype, with higher concentrations of TNF- $\alpha$ and lower concentrations of IL-4 in the serum following $\mathrm{rTe}$ $\beta$-tubulin immunization in mice. Immunized mice demonstrated a progressive drop in serum IL-5 and IL-10 concentrations following challenge. Serum IFN- $\gamma$ concentration increased in $\mathrm{rTe}$ - $\beta$-tubulin-immunized mice but dropped after 3 days post challenge. Such serum cytokine profiles suggest a strongly Th1 polarized immune response. Mice immunized with $\mathrm{r} T e-\beta$-tubulin also showed falling TNF- $\alpha$ serum concentrations following a brief rise immediately post challenge, suggesting better protection and reduced pathological changes. These results suggest that $\mathrm{rTe}-\beta$-tubulin immunization promotes a Th1-biased recall response following $T$. evansi challenge. Such a Th1 polarized response has been shown to be pivotal for protection against T. evansi infections $[20-23,31]$ as was demonstrated by the longer survival of vaccinates in the present study.

Although the role of adaptive immunity in protection against extracellular pathogens such as trypanosomes are well recognized [32, 33], the independent contributions of the cell-mediated or humoral immune mechanisms are less well understood. Profiles of induced 
cytokines are, in general, good indicators of the nature of immune responses mounted by the host against a pathogen and may aid in predicting the outcome of infection. Although the levels of various cytokines have served as reliable indicators of the impact of a pathogen on a host, studies [34-36] have shown repeatedly that attempting to predict the outcome of an infection based on a single cytokine response is unlikely to be successful. For example, IFN- $\gamma$, an important cytokine of the Th1 subset, activates and stimulates the macrophages to produce IL-12 that not only helps in differentiation of Th1 cells, but also inhibits the expansion of Th2 type T-cell population. IFN- $\gamma$ also induces class switching to IgG2a [37] and is a hallmark of humoral immunity to trypanosomosis in various natural and experimental hosts $[20-23,31]$. Mice immunized with $\mathrm{rTe}-\beta$-tubulin showed higher serum levels of IFN $\gamma$ following $T$. evansi challenge compared to mice immunized with the adjuvant alone. In the present study, elevated IFN- $\gamma$ levels may have contributed to the better control of $T$. evansi parasitemia observed in the immunized compared to sham-immunized mice. However, enhanced production of IFN- $\gamma$ has also been correlated with early mortality in mice infected with $T$. congolense or $T$. brucei $[38,39]$, primarily owing to the systemic inflammatory response syndrome (SIRS). This was further substantiated by the observation that anti-IFN $\gamma$ antibodies could prevent early mortality in these infections [40].

The ability of elevated IFNY levels following virulent challenge with $T$. evansi to both aid control of parasitemia and simultaneously exacerbate damage to the host may be explained by the varied roles that IFN- $\gamma$ plays in the host. Barkhuizen et al. [41] showed that IFN- $\gamma$ plays a central role in resistance against trypanosomosis by inducing macrophages to generate trypanocidal molecules such as reactive oxygen intermediates, reactive nitrogen intermediates or TNF- $\alpha$. Although TNF- $\alpha$ is vital for resistance against trypanosomosis [31], increasing TNF- $\alpha$ levels indicate a failing immune system and an uncontained infection [42-44]. Additionally, it has been challenging to determine if an individual cytokine response is a consequence of an infection or exacerbates one. For example, a surge in IL-10 in serum often follows release of pro-inflammatory cytokines such IFN- $\gamma$ during an infection; in such cases the surge in IL-10 production is a consequence of inflammation rather than a contributor to it [45]. The cytokines IL-4, IL-5 and IL-10 are thought to balance the pro-inflammatory milieu generated in an infection to keep the host tissue safe from its own inflammatory immune responses. In the protected mice, we observed a spike in serum IFN- $\gamma$ levels concurrently with lower IL-4 and IL-5 levels immediately after challenge. This IFN- $\gamma$ surge is followed by a sudden decrease, most likely associated with a control of the infection, as indicated by a corresponding drop of IL-10 and TNF- $\alpha$ concentration in the serum. Although it may be difficult to predict the outcome of an infection from the levels and patterns of individual serum cytokine responses, deducing overall cytokine profiles by measuring multiple, interacting cytokines helps in predicting the course of the infection. This knowledge might also help to define a desirable protective cytokine profile indicative of successful vaccination.

\section{Conclusions}

Mice immunized with $\mathrm{rTe}$ - $\beta$-tubulin in this study showed a predominant Th1 polarization in the elicited immune response as evidenced by both the cytokine profile and the IgG2a isotype of circulating anti-Te- $\beta$ tubulin antibodies generated in immunized mice. Increased resistance to trypanosomosis in mice may be correlated with higher serum level of the parasite specific IgG2a antibodies; the elevated serum titers of antitubulin IgG2a class antibodies post-immunization likely contributed to our observed protection of mice from the lethal challenge. This study reasserts the potential of prima facie concealed antigens in eliciting protective humoral immune responses against pathogens; such cryptic antigens may be crucial resources in the fight against complex eukaryotic pathogens and their complicated biologies.

\section{Abbreviations}

${ }^{\circ} \mathrm{C}$ : Degree celsius; DPC: Day post challenge; ELISA: Enzyme linked immunosorbent assay; FACS: Fluorescence activated cell sorting;

FCA: Freund's complete adjuvant; H: Hour; IFA: Freund's incomplete adjuvant;

IFN-Y: Interferon gamma; IgG2a: Immunoglobulin G 2a isotype; IL-

10: Interleukin 10; IL-4: Interleukin 4; IL-5: Interleukin 5; ml: Milliliter;

PCR: Polymerase chain reaction; SDS-PAGE: Sodium dodecyl sulphate polyacrylamide gel electrophoresis; Te- $\beta$-tubulin: Trypanosoma evansi beta tubulin; Th1: T-helper cell type 1; Th2: T-helper cell type 2; TNF-a: Tumor necrosis factor alpha; $\mu \mathrm{g}$ : Microgram; $\mu$ l: Microliter.

\section{Competing interests}

The authors declare that they have no competing interests.

\section{Authors' contributions}

AKT and SPK conceived of the study, participated in the design of the study, carried out the experimental work and analyses, and drafted the manuscript. JRB assisted in data analyses and helped to draft the manuscript. SB was associated with isolation and propagation of $T$. evansi and was involved in the in vivo experiments. BS assisted in generation of data using FACS. All authors read and approved the final manuscript.

\section{Acknowledgements}

The authors acknowledge the Director, Indian Veterinary Research Institute, Izatnagar for the facilities provided to undertake the study.

\section{Author details}

'Department of Pathobiology, Ontario Veterinary College, University of Guelph, Guelph, ON N1G 2W1, Canada. ${ }^{2}$ Division of Parasitology, Indian Veterinary Research Institute, Izatnagar, Uttar Pradesh 243 122, India.

${ }^{3}$ Department of Microbiology, University of lowa Carver College of Medicine, Iowa City, IA 52242, USA. ${ }^{4}$ Division of Animal Biochemistry, Indian Veterinary Research Institute, Izatnagar, Uttar Pradesh 243 122, India. ${ }^{5}$ Department of Parasitology, West Bengal University of Animal and Fishery Sciences, 37 \& 68, Kshudiram Bose Sarani, Belgachia, Kolkata, West Bengal 700037, India. 
Received: 26 August 2015 Accepted: 30 November 2015

\section{Published online: 09 November 2015}

\section{References}

1. Luckins AG. Effect of concurrent infections with Trypanosoma congolense in rabbits on the development of immunity after trypanocidal drug treatments. Ann Trop Med Parasitol. 1986;80:131-3.

2. Lun ZR, Fang Y, Wang CJ, Brun R. Trypanosomiasis of domestic animals in China. Parasitol Today. 1993;9:41-5.

3. Gutierrez C, Desquesnes M, Touratier L, Büscher P. Trypanosoma evansi: recent outbreaks in Europe. Vet Parasitol. 2010;174:26-9.

4. Kundu K, Tewari AK, Kurup SP, Baidya S, Rao JR, Joshi P. Sero-surveillance for surra in cattle using native surface glycoprotein antigen from Trypanosoma evansi. Vet Parasitol. 2013;196:258-64.

5. Bajyana Songa E, Kageruka P, Hamers R. The use of the card agglutination test (Testryp CATT) for the serodiagnosis of T. evansi infection. Ann Soc Belg Med Trop. 1987;67:51-7.

6. Taylor KA. Immune responses of cattle to African trypanosomes: protective or pathogenic? Int J Parasitol. 1998:28:219-40.

7. Holland WG, Do TT, Huong NT, Dung NT, Thanh NG, Vercruysse J, et al. The effect of Trypanosoma evansi infection on pig performance and vaccination against classical swine fever. Vet Parasitol. 2003;111:115-23.

8. Desquesnes M, Dargantes A, Lai DH, Lun ZR, Holzmuller P, Jittapalapong S. Trypanosoma evansi and surra: a review and perspectives on transmission, epidemiology and control, impact, and zoonotic aspects. Biomed Res Int. 2013;Article ID 321237:20

9. Lubega GW, Byarugaba DK, Prichard RK. Immunization with a tubulin-rich preparation from Trypanosoma brucei confers broad protection against African trypanosomosis. Exp Parasitol. 2002;102:9-22.

10. Li SQ, Fung MC, Reid SA, Inoue N, Lun ZR. Immunization with recombinant beta-tubulin from Trypanosoma evansi induced protection against T. evansi, T. equiperdum and T. b. brucei infection in mice. Parasite Immunol. 2007:29:191-9.

11. Overath $P$, Chaudhri M, Steverding D, Ziegelbauer K. Invariant surface proteins in bloodstream forms of Trypanosoma brucei. Parasitol Today. 1994;10:53-8.

12. Li SQ, Yang WB, Ma LJ, Xi SM, Chen QL, Song XW, et al. Immunization with recombinant actin from Trypanosoma evansi induces protective immunity against T. evansi, T. equiperdum and T. b. brucei infection. Parasitol Res. 2009;104:429-35.

13. Silva MS, Prazeres DM, Lanca A, Atouguia J, Monteiro GA. Trans-sialidase from Trypanosoma brucei as a potential target for DNA vaccine development against African trypanosomiasis. Parasitol Res. 2009;105:1223-9.

14. La Greca F, Magez S. Vaccination against trypanosomiasis: Can it be done or is the trypanosome truly the ultimate immune destroyer and escape artist? Hum Vaccines. 2011;7:1225-33.

15. Certa U, Ghersa P, Dobeli H, Matile H, Kocher HP, Shrivastava IK, et al. Aldolase activity of a Plasmodium falciparum protein with protective properties. Science. 1998;240:1036-8.

16. Waine GJ, Becker MM, Scott JC, Kalinna BH, Yang W, McManus DP Purification of a recombinant Schistosoma japonicum antigen homologous to the 22-kDa membrane-associated antigen of S. mansoni, a putative vaccine candidate against schistosomiasis. Gene. 1994;142:259-63.

17. Kurup SP, Tewari AK. Induction of protective immune response in mice by a DNA vaccine encoding Trypanosoma evansi beta tubulin gene. Vet Parasitol. 2012;187:9-16.

18. Dempsey WL, Mansfield JM. Lymphocyte function in experimental African trypanosomiasis. V. Role of antibody and the mononuclear phagocyte system in variant-specific immunity. J Immunol. 1983;130:405-11.

19. Reinitz DM, Mansfield JM. T-cell-independent and T-cell-dependent B-cell responses to exposed variant surface glycoprotein epitopes in trypanosome-infected mice. Infect Immun. 1990;58:2337-42

20. Mansfield JM. T-cell responses to the trypanosome variant surface glycoprotein: a new paradigm? Parasitol Today. 1994;10:267-70.

21. Schopf LR, Filutowicz H, Bi XJ, Mansfield JM. Interleukin-4-dependent immunoglobulin G1 isotype switch in the presence of a polarized antigenspecific Th1-cell response to the trypanosome variant surface glycoprotein. Infect Immun. 1998;66:451-61.

22. Liu Y, Ragaa E, Li Z, Nuortio L, Mustafa A, Bakhiet M. Interferon-gamma and interleukin-12 genes are preferentially expressed during early experimental
African trypanosomiasis and suppressed by denervation of the spleen. Scand J Immunol. 1999;50:485-91.

23. Magez S, Stijlemans B, Baral T, De Baetselier P. VSG-GPI anchors of African trypanosomes: Their role in macrophage activation and induction of infection-associated immunopathology. Microbes Infect. 2002;4:999-1006.

24. Cross GA. Cellular and genetic aspects of antigenic variation in trypanosomes. Annu Rev Immunol. 1990;8:83-110.

25. Naessens J. Bovine trypanotolerance: A natural ability to prevent severe anaemia and haemophagocytic syndrome? Int J Parasitol. 2006;36:521-8.

26. Vercruysse J, Schetters TPM, Knox DP, Willadsen P, Claerebout E. Control of parasitic disease using vaccines: An answer to drug resistance? Rev Sci Tech Off Int Epiz. 2007;2007(26):105-15.

27. Meeusen ENT, Walker J, Peters A, Pastoret P, Jungersen G. Current Status of Veterinary Vaccines. Clin Microbiol Rev. 2007;2007(20):489-510.

28. Magez S. Current status of vaccination against African trypanosomiasis. Parasitology. 2010;137:2017-27.

29. Balaban N, Waithaka HK, Njogu AR, Goldman R. Intracellular antigens (microtubule-associated protein copurified with glycosomal enzymes)-possible vaccines against trypanosomiasis. J Infect Dis. 1995; 172:845-50.

30. Shibaki A, Katz SI. Induction of skewed Th1/Th2 T-cell differentiation via subcutaneous immunization with Freund's adjuvant. Exp Dermatol. 2002;11:126-34.

31. Magez S, Radwanska M, Beschin A, Sekikawa K, De Baetselier P. Tumor necrosis factor alpha is a key mediator in the regulation of experimental Trypanosoma brucei infections. Infect Immun. 1999;67:3128-32.

32. Namangala B. How the African trypanosomes evade host immune killing Parasite Immunol. 2011:33:430-7.

33. Mansfield JM, Paulnock DM, Hedberg GM. Bridging Innate and Adaptive Immunity in African Trypanosomiasis in Trypanosomes and Trypanosomiasis, (S. Magez, S. and M. Radwanska, M. (eds.)), Vienna, Austria: Springer-Verlag Wien; 2014, pp 89-114.

34. Stijlemans B, Guilliams M, Raes G, Beschin A, Magez S, De Baetselier P. African trypanosomiasis: from immune escape and immunopathology to immune intervention. Vet Parasitol. 2007;148:3-13.

35. Tabel H, Wei G, Shi M. T cells and experimental immunopathogenesis of African trypanosomiasis. Immunol Rev. 2008;225:128-39.

36. Miller CMD, Smith NC, Ikin RJ, Boulter NR, Dalton JP, Donnelly S. Immunological Interactions between 2 common pathogens, Th1-inducing protozoan Toxoplasma gondii and the Th2-Inducing helminth Fasciola hepatica. PLoS ONE. 2009;4:e5692

37. Yoshimoto T, Okada K, Morishima N, Kamiya S, Owaki T, Asakawa M, et al. Induction of IgG2a class switching in B cells by IL-27. J Immunol. 2004;173:2479-85.

38. Shi M, Pan W, Tabel H. Experimental African trypanosomiasis: IFN- $\gamma$ mediates early mortality. Eur J Immunol. 2003;33:108-18.

39. Shi M, Wei G, Pan W, Tabel H. Experimental African trypanosomiasis: A subset of pathogenic, IFN-gamma-producing, MHC class II-restricted CD4+ T cells mediates early mortality in highly susceptible mice. J Immunol. 2006;176:1724-32.

40. Uzonna JE, Kaushik RS, Gordon JR, Tabel H. Experimental murine Trypanosoma congolense infections. I. Administration of anti-IFN-gamma antibodies alters trypanosome-susceptible mice to a resistant-like phenotype. J Immunol. 1998;161:5507-15.

41. Barkhuizen M, Magez S, Atkinson RA, Brombacher F. Interleukin-12p70dependent interferon- gamma production is crucial for resistance in African trypanosomiasis. J Infect Dis. 2007;196:1253-60.

42. Starobinas N, Russo M, Minoprio P, Hontebeyrie-Joskowicz M. Is TNF alpha involved in early susceptibility of Trypanosoma cruzi-infected $\mathrm{C} 3 \mathrm{H} / \mathrm{He}$ mice? Res Immunol. 1991;142:117-22.

43. Okomo-Assoumou MC, Geffard M, Daulouede S, Chaugier C, Lemesre JL, Vincendeau P. Circulating antibodies directed against tryptophan-like epitopes in sera of patients with human African trypanosomiasis. Am J Trop Med Hyg. 1995;52:461-7.

44. Andrade Jr DR, Santos SA, Castro I, Andrade DR. Correlation between serum tumor necrosis factor alpha levels and clinical severity of tuberculosis. Braz J Infect Dis. 2008;12:226-33.

45. Couper KN, Blount DG, Wilson MS, Hafalla JC, Belkaid Y, Kamanaka M, et al. IL-10 from CD4CD25Foxp3CD127 adaptive regulatory T cells modulates parasite clearance and pathology during malaria infection. PLoS Pathog. 2008;4:e1000004 